${ }^{1} \mathrm{M}$ Pritchard, ${ }^{2} \mathrm{~T}$ de Dassel, ${ }^{2} \mathrm{D}$ Cartwright, ${ }^{3} \mathrm{~F}$ Bogossian, ${ }^{4} \mathrm{~J}$ Paynter, ${ }^{5} \mathrm{E}$ Beller, ${ }^{6} \mathrm{~S}$ Russo, ${ }^{7} \mathrm{C}$ Crothers, ${ }^{8} \mathrm{~L}$ Johnston, ${ }^{9} \mathrm{~J}$ Scott. ${ }^{1}$ Perinatal Centre for Clinical Research, The University of Queensland Royal Brisbane Women's Hospital, Brisbane, Australia; ${ }^{2}$ Psychlogy, Royal Brisbane Women's Hospital, Brisbane, Australia; ${ }^{3}$ Nursing, The University of Queensland, Brisbane, Australia; ${ }^{4}$ Psychology, Autism Early Intervention Outcomes Unit, Brisbane, Australia; ${ }^{5}$ Centre for Research in Evidence-Based Practice, Bond University, Robina, Australia; ${ }^{6}$ Psychology, Better Life Psychology, Brisbane, Australia; ${ }^{7}$ Perinatal, The University of Queensland, Brisbane, Australia; ${ }^{8}$ Nursing, Queens University, Belfast, Ireland; ${ }^{9}$ Centre for Clinical Research, The University of Queensland, Brisbane, Australia

\subsection{6/archdischild-2014-307384.128}

The Autism Diagnostic Observation Schedule (ADOS) is the only available semi-structured observational assessment of autism and has not been used extensively in preterm toddlers. We used a screening and diagnostic method to determine the diagnostic prevalence of autism and the characteristics of screen positive cases.

Methods A 12-month birth-cohort of toddlers (2-and-4-years-old) born with a gestational age $<29$-weeks were administered the Modified Checklist of Autism in Toddlers (M-CHAT)-and follow-up interview $\left(\mathrm{FI}^{\mathrm{TM}}\right)$ screen, the ADOS and neurodevelopmental assessments (Bayley Scales of Infant and Toddler Development III-2-yearolds, Wechsler Preschool and Primary Scale of Intelligence-III and Adaptive Behaviour Assessment System-Second Edition-4-year-olds). The ADOS was conducted on toddlers with M-CHAT-FI ${ }^{\mathrm{TM}}$ positive screens.

Results Complete data were available on 88\% (169/192) of children. Thirteen-percent screened M-CHAT-FI ${ }^{\mathrm{TM}}$ positive. ADOS (DSM-IV-criteria) classified 1.8\% with autistic disorder and none with ASD. Multivariate analysis showed that social emotional delay $(\mathrm{p}=<0.001$ ) was the only neurodevelopmental factor independently associated with M-CHAT-FI ${ }^{\mathrm{TM}}$ positive screens even after adjusting for psychosocial risk and child gender. All but one of the M-CHAT-FI ${ }^{\mathrm{TM}}$ positive screens scored some level of abnormality at the domain and item level on the ADOS testing, one third of these reached the Communication Total category cut off for ASD for the combined Communication and Social Interaction Total Score. Four children displayed comorbid atypical development (DQ/IQ <-2SD).

Conclusion The ADOS reports a lower incidence of autistic disorder in children born very preterm compared to studies using diagnostic interview based tools. The distinguishing features of the M-CHAT-FI ${ }^{\mathrm{TM}}$ positive cases suggest a sub-threshold communication dysfunction profile.

\section{0-061 CELL-BASED THERAPY FOR HYPOXIC-ISCHAEMIC INJURY IN THE PRETERM BRAIN}

${ }^{1}$ RK Jellema, ${ }^{2}$ TGAM Wolfs, ${ }^{3} \mathrm{~V}$ Lima Passos, ${ }^{4} \mathrm{~A}$ Zwanenburg, ${ }^{1} \mathrm{DRMG}$ Ophelders, ${ }^{1}$ E Kuypers, ${ }^{5}$ AHN Hopman, ${ }^{6}$ J Dudink, ${ }^{7}$ HW Steinbusch, ${ }^{8} \mathrm{P}$ Andriessen, ${ }^{9}$ WTV Germeraad, ${ }^{10} \mathrm{~J}$ Vanderlocht, ${ }^{11} \mathrm{BW}$ Kramer. ${ }^{1}$ Department of Pediatrics. School for Mental Health and Neuroscience, Maastricht University, Maastricht, Netherlands; ${ }^{2}$ Department of Pediatrics. School of Oncology and Developmental Biology, Maastricht University, Maastricht, Netherlands; ${ }^{3}$ Department of Methodology \& Statistics, Maastricht University, Maastricht, Netherlands; ${ }^{4}$ Department of Pediatrics. Department of Biomedical Engineering, Maastricht University, Maastricht, Netherlands; ${ }^{5}$ Department of Molecular Cell Biology. School of Oncology and Developmental Biology, Maastricht University, Maastricht, Netherlands; ${ }^{6}$ Department of Neonatology and Neuroscience, Sophia Children's Hospital, Rotterdam, Netherlands; 'School for Mental Health and Neuroscience, Maastricht University, Maastricht, Netherlands, ${ }^{8}$ Department of Pediatrics, Máxima Medical Centre, Veldhoven, Netherlands; ${ }^{9}$ Department of Internal Medicine Division of Haematology.
School of Oncology and Developmental Biology, Maastricht University Medical Center, Maastricht, Netherlands; ${ }^{10}$ Department of Transplantation Immunology Tissue Typing Laboratory. School of Oncology and Developmental Biology, Maastricht University Medical Center, Maastricht, Netherlands; ${ }^{11}$ Department of Pediatrics. School for Mental Health and Neuroscience. School of Oncology and Developmental Biology, Maastricht University, Maastricht, Netherlands

\subsection{6/archdischild-2014-307384.129}

Background and aims Preterm infants are prone to hypoxicischaemic encephalopathy. No therapy exists to treat this brain injury. The objective of this study was to assess the neuroprotective effect of exogenous administration of stem cells and the mobilisation of endogenous stem cells in the ovine preterm brain after global hypoxia-ischemia.

Methods Instrumented preterm sheep were subjected to global hypoxia-ischemia by $25 \mathrm{~min}$ of umbilical cord occlusion at a gestational age of 104 (term is 150) days. During a 7 day reperfusion period all vital parameters, including (amplitude-integrated) electroencephalogram, were recorded. At the end of the experiment, the preterm brain was assessed by histology and diffusion tensor imaging (DTI).

Results Systemic administration of exogenous mesenchymal stem cells (MSCs) reduced cerebral inflammation (i.e. microglia proliferation) and white matter injury. MSCs induced T-cell tolerance, which was paralleled with diminished mobilisation and invasion of these cells in the preterm brain. In addition, MSCs decreased number of seizures after global hypoxia-ischemia, indicating functional improvement.

Similarly, mobilisation of endogenous stem cells using systemic granulocyte-colony stimulating factor (G-CSF) reduced cerebral inflammation and white matter injury. However, G-CSF did not reduce the number of seizures after global hypoxiaischemia.

Conclusion We have shown for the first time in a translational animal model that cell-based therapy is effective in protecting the preterm brain against the cerebral and peripheral inflammatory responses which are involved in the aetiology of white matter injury in the preterm brain after global hypoxia-ischemia. Our studies form the basis for future clinical trials studying feasibility of cell-based therapy in preterm infants with hypoxicischaemic encephalopathy.

\section{Neonatal Brain and Development - Evolving Techniques}

\section{0-062 EARLY BRAIN ACTIVITY AND CORTICAL DEVELOPMENT IN PRETERM INFANTS}

${ }^{1} \mathrm{ML}$ Tataranno, ${ }^{2} \mathrm{P}$ Moeskops, ${ }^{3} \mathrm{LS}$ de Vries, ${ }^{3} \mathrm{KJ}$ Kersbergen, ${ }^{3} \mathrm{~F}$ Groenendaal, ${ }^{3} \mathrm{LGM}$ van Rooij, ${ }^{3} \mathrm{MC}$ Toet, ${ }^{1} \mathrm{G}$ Buonocore, ${ }^{2} \mathrm{I}$ Isgum, ${ }^{3} \mathrm{MJNL}$ Benders. ${ }^{1}$ Department of Molecular and Developmental Medicine, University of Siena, Siena, Italy; ${ }^{2}$ Image Sciences Institute, University Medical Center Utrecht, Utrecht, Netherlands; ${ }^{3}$ Department of Neonatology Division of Perinatology, University Medical Center Utrecht, Utrecht, Netherlands

\subsection{6/archdischild-2014-307384.130}

Background and aim Early brain activity is crucial for neuronal growth. It is well known that the cerebral cortex develops rapidly in the last trimester of pregnancy. We investigated whether early brain activity was related to the rate of cortical 